\title{
Functional Genetic Variant in ATG5 Gene Promoter in Acute Myocardial Infarction
}

\author{
Yexin Zhang, ${ }^{1}$ Xiaohui He, ${ }^{1}$ Jiarui Li, ${ }^{1}$ Wentao Yang, ${ }^{1}$ Yinghua Cui, ${ }^{2}$ Shuchao Pang, \\ Haihua Wang $\mathbb{D}^{3},{ }^{3}$ and Bo Yan $\mathbb{D}^{3,4,5}$ \\ ${ }^{1}$ Department of Medicine, Shandong University School of Medicine, Jinan, Shandong 250012, China \\ ${ }^{2}$ Division of Cardiology, Affiliated Hospital of Jining Medical University, Jining Medical University, Jining, \\ Shandong 272029, China \\ ${ }^{3}$ Shandong Provincial Key Laboratory of Cardiac Disease Diagnosis and Treatment, \\ Affiliated Hospital of Jining Medical University, Jining Medical University, Jining, Shandong 272029, China \\ ${ }^{4}$ The Center for Molecular Genetics of Cardiovascular Diseases, Affiliated Hospital of Jining Medical University, \\ Jining Medical University, Jining, Shandong 272029, China \\ ${ }^{5}$ Shandong Provincial Sino-US Cooperation Research Center for Translational Medicine, \\ Affiliated Hospital of Jining Medical University, Jining Medical University, Jining, Shandong 272029, China
}

Correspondence should be addressed to Haihua Wang; whh__wz@126.com and Bo Yan; yanbo@mail.jnmc.edu.cn

Received 25 August 2019; Revised 21 February 2020; Accepted 2 March 2020; Published 21 April 2020

Academic Editor: Anne Knowlton

Copyright (C) 2020 Yexin Zhang et al. This is an open access article distributed under the Creative Commons Attribution License, which permits unrestricted use, distribution, and reproduction in any medium, provided the original work is properly cited.

Coronary artery disease (CAD) including acute myocardial infarction (AMI) is an inflammatory and metabolic disease mainly caused by atherosclerosis. Dysfunctional autophagy has been associated with abnormal lipid metabolism and inflammation. In previous studies, we have reported altered autophagic activity in AMI patients. As autophagy-related protein 5 (ATG5) is a core protein in autophagy, we speculated that altered ATG5 level may contribute to CAD and AMI development. In this study, the promoter of the ATG5 gene was genetically and functionally investigated in large groups of AMI patients $(n=378)$ and ethnicmatched healthy controls $(n=386)$. The results showed that a total of 15 genetic variants including 6 single-nucleotide polymorphisms (SNPs) in the ATG5 gene promoter were found in this study population. A novel deletion variant (g.106326168_70delTCT) and an SNP [g.106325757C > G (rs190825454)] were found in one 66-year-old male patient with nonST-segment elevated AMI, but in none of controls. In cultured HEK-293 and H9c2 cells, the deletion variant significantly decreased the transcriptional activity of the ATG5 gene promoter $(P<0.01)$. In contrast, the genetic variants either identified only in controls or found in both AMI patients and controls did not affect the transcriptional activity of the ATG5 gene promoter $(P>0.05)$. Furthermore, an electrophoretic mobility shift assay showed that the deletion variant evidently affected the binding of a transcription factor. Therefore, the genetic variant identified in AMI may affect the activity of the ATG5 gene promoter and change the ATG5 level, contributing to AMI as a rare risk factor.

\section{Introduction}

Coronary artery disease (CAD) including acute myocardial infarction (AMI) is an inflammatory and metabolic disease, which is mainly caused by atherosclerosis. Abnormal lipid metabolism and inflammation play critical roles in the initiation and progression of atherosclerosis and its complications $[1,2]$. Although genome-wide association studies have identified more than 60 genetic loci for CAD and AMI, these collective genetic loci could explain only $<10 \%$ of cases [3]. Therefore, genetic causes and molecular mechanisms for CAD and AMI remain to be investigated and elucidated.

Autophagy is an evolutionally conserved process in cells to deliver cytoplasmic contents to lysosomes for degradation. Human studies and animal experiments have demonstrated that autophagy plays essential roles in many physiological processes, such as maintaining cellular homeostasis, regulating cell death and survival, aging, lipid 
metabolism, immune response, and inflammation [4-6]. Dysfunctional autophagy has been implicated in a wide range of human diseases, including neurodegenerative diseases, metabolic syndrome, cardiovascular diseases, and cancers $[7,8]$. In the cardiovascular system, autophagy functions in all cell types and is essential to preserve cardiovascular structure and function under baseline conditions [9]. As optimal autophagic activity is critical to cardiovascular homeostasis and function, excessive or insufficient levels of autophagic flux may contribute to the development of cardiovascular diseases, such as atherosclerosis, cardiomyopathy, $\mathrm{CAD}$, and $\mathrm{AMI}[7,10]$.

To date, more than 40 autophagy-related (ATG) proteins have been identified and ATG5 is a critical core protein for autophagy [11]. During the process of autophagosome formation, ATG5 is first formed, a conjugate with ATG12, by the activation of ATG7 (E1-like enzyme) and ATG10 (E2like enzyme). The ATG5-ATG12 conjugate then binds to membrane-associated ATG16L, thereby forming the ATG16L complex. The ATG5-ATG12-ATG16L complex functions as an E3 ligase to facilitate the covalent conjugation of microtubule-associated protein light chain 3 (LC3)-phosphatidylethanolamine [12-14]. Mice deficient for ATG5 appear almost normal at birth but die within 1 day of delivery [15]. Mice with cardiac-specific ATG5 deletion are born normally and exhibit significant cardiac dysfunction at the age of 10 months $[16,17]$. In contrast, ubiquitous overexpression of ATG5 extends median lifespan of mice [18]. Moreover, ATG5 deficiency-mediated autophagy contributes to cardiac inflammation and injury by increasing $\mathrm{NF}-\kappa \mathrm{B}$ activity in macrophages [19]. In mice with a macrophage-specific ablation of ATG5, P62 deficiency increases atherosclerotic plaque burden, indicating ATG5 involvement in atherosclerosis formation [20].

We have previously reported altered LC3 gene expression levels in CAD and AMI patients [21]. Several genetic variants have been identified in the ATG7 and LC3 gene in AMI patients [22, 23]. As ATG5 is one of core proteins for autophagy, we postulated that dysregulated ATG5 gene expression may lead to altered autophagic activity, playing an important role in the development of CAD and AMI. In this study, we genetically and functionally analyzed the promoter of the ATG5 gene in a large cohort of AMI patients and ethnic-matched healthy controls.

\section{Materials and Methods}

2.1. Study Population. AMI patients $(n=379$, male 283, female 96 , average age 60.43 years) were recruited from the Division of Cardiology, Affiliated Hospital of Jining Medical University (Jining, Shandong Province, China) during the period of April 2016 to April 2018. All AMI patients were diagnosed with clinical manifestations, electrocardiogram, and three-dimensional echocardiography. Ethnic-matched healthy controls $(n=386$, male 198 , female 188 , median age 51.32 years) were from the Physical Examination Center in the same hospital. Controls with CAD family history were excluded from this study. This study was approved by the Human Ethic Committee, Affiliated Hospital of Jining
Medical University, and conducted according to the principles of the Declaration of Helsinki. Informed consent was obtained from all participants.

2.2. Direct DNA Sequencing. Genomic DNAs were purified from peripheral blood leukocytes as previously described $[22,23]$. The ATG5 gene promoter region (from $-1069 \mathrm{bp}$ to $+103 \mathrm{bp}$ to the transcription start site) was sequenced and analyzed. Two overlapped DNA fragments covering the ATG5 gene promoter, $649 \mathrm{bp}(-1069 \mathrm{bp} \sim-421 \mathrm{bp})$ and 658 bp $(-655 \mathrm{bp} \sim+103 \mathrm{bp})$, were generated by PCR. PCR primers were designed based on genomic sequence of the human ATG5 gene (NCBI, NC_000006.12) (Table 1). PCR products were bidirectionally sequenced with an Applied Biosystems 3500XL genetic analyzer. The sequences were aligned and compared with the wild-type ATG5 gene promoter, and genetic variants were identified.

2.3. Functional Analysis with Dual-Luciferase Reporter Assay. Report gene expression vectors with wild-type and variant ATG5 gene promoters were constructed with a firefly luciferase reporter vector (pGL3-basic). PCR primers to generate the ATG5 gene promoter are shown in Table 1. All expression vectors were further confirmed with sequencing. Designated expression vectors were then transfected into cultured cells (human embryonic kidney cells HEK-293 and rat cardiomyocyte cells $\mathrm{H} 9 \mathrm{c} 2$ ), and dual-luciferase activities were examined. The experimental details were previously described [22, 23]. Transcriptional acitivity of the wild-type ATG5 gene promoter was designed as $100 \%$. Relative activity of the variant ATG5 gene promoter was calculated. All the experiments were repeated three times independently, in triplicate.

2.4. Electrophoretic Mobility Shift Assay. Nuclear extract preparation and electrophoretic mobility shift assay (EMSA) was previously described [22, 23]. Nuclear extracts were prepared from HEK-293 and H9c2 cells. Double-stranded biotinylated oligonucleotides (30 bp) included wild-type $\left(5^{\prime}\right.$ TCCAACAAAGTAGAGAAGAAGATCAAATAA-3 ${ }^{\prime}$ ) and genetic variant g.106326168_70delTCT (5'-TCCAACAAAGTAGAGAAGATCAAATAAGAA-3').

2.5. Statistical Analysis. Quantitative data were expressed as mean \pm SEM and analyzed by a standard Student's $t$-test. Frequencies of genetic variants in AMI patients and controls were analyzed and compared with SPSS v23.0. $P<0.05$ was considered as statistically significant.

\section{Results}

3.1. Genetic Variants Identified in AMI Patients and Controls. A total of 15 genetic variants, including 6 single-nucleotide polymorphisms (SNPs), were identified in this study. Locations and frequencies of the genetic variants are depicted in Figure 1 and summarized in Table 2. A novel deletion variant (g.106326168_70delTCT) and an SNP 
TABLE 1: PCR primers for the human ATG5 gene promoter.

\begin{tabular}{lccc}
\hline PCR primers & DNA sequences & Location & Position \\
\hline Sequencing & & & Products \\
ATG5-F1 & $5^{\prime}$-GGCATGCTTCCCTAACTTGA-3' & 106326889 & $-1069 \mathrm{bp}$ \\
ATG5-R1 & $5^{\prime}$-CCCACCCATCCAAGAGTACA-3' & 106326241 & $-421 \mathrm{bp}$ \\
ATG5-F2 & $5^{\prime}$-TCTCGATCTCCTGACCTCGT-3' & 106326375 & $-655 \mathrm{bp}$ \\
ATG5-R2 & $5^{\prime}$-CACTTCCGCCCTCTGGTAT-3' & 106325718 & $+103 \mathrm{bp}$ \\
Functioning & & & $658 \mathrm{bp}$ \\
ATG5-F & $5^{\prime}$-(KpnI)-GGCATGCTTCCCTAACTTGA-3' & 106326889 & $-1069 \mathrm{bp}$ \\
ATG5-R & $5^{\prime}$-(HindIII)-CACTTCCGCCCTCTGGTAT-3' & 106325718 & $+103 \mathrm{bp}$ \\
\hline
\end{tabular}

PCR primers are designed based on the genomic DNA sequence of the ATG5 gene (NC_000006.12). The transcription start site is at the position of 106325820 $(+1)$.

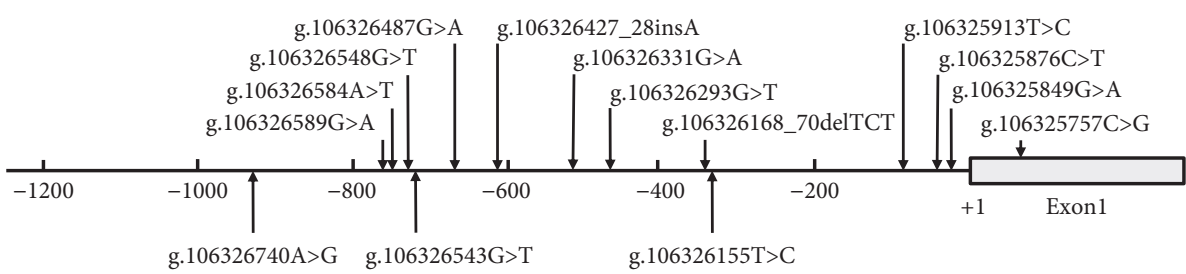

FIGURE 1: Locations and sequencing chromatograms of the genetic variants in the ATG5 gene promoter. The numbers represent the genomic DNA sequences of the human ATG5 gene (Genbank accession number NC_000006.12) upstream to the transcription start site (at the position of 106325820), which is set as +1 .

TABLE 2: Genetic variants in ATG5 gene promoters in AMI patients and controls.

\begin{tabular}{|c|c|c|c|c|c|}
\hline Genetic variants & Genotypes & Location $^{1}$ & Controls $(n=386)$ & AMI $(n=378)$ & $P$ value \\
\hline g.106326740A > G & $\mathrm{TC}$ & $-920 b p$ & 2 & 3 & 1.000 \\
\hline \multirow[t]{3}{*}{ g.106326589G > A (rs506027) } & GG & $-769 b p$ & 83 & 78 & 0.883 \\
\hline & GA & & 177 & 180 & \\
\hline & AA & & 126 & 120 & \\
\hline g.106326584A > T & AT & $-764 b p$ & 3 & 5 & 1.000 \\
\hline g.106326548G > T (rs117781908) & GT & $-728 \mathrm{bp}$ & 1 & 0 & - \\
\hline g.106326543G > T (rs182877945) & GT & $-723 b p$ & 1 & 0 & - \\
\hline g.106326487G > A & GA & $-667 \mathrm{bp}$ & 1 & 2 & 1.000 \\
\hline g.106326427_28insA & $-/ A$ & $-607 \mathrm{bp}$ & 1 & 1 & 1.000 \\
\hline g.106326331G $>$ A & GA & $-511 b p$ & 1 & 2 & 1.000 \\
\hline g.106326293G > T (rs560549742) & GT & $-473 b p$ & 1 & 0 & - \\
\hline g.106326168_70delTCT & TCT/- & $-348 \mathrm{bp}$ & 0 & 1 & - \\
\hline \multirow[t]{3}{*}{ g.106326155T > C (rs510432) } & TT & $-335 b p$ & 83 & 78 & 0.883 \\
\hline & $\mathrm{TC}$ & & 177 & 180 & \\
\hline & $\mathrm{CC}$ & & 126 & 120 & \\
\hline g.106325913T > C & $\mathrm{TC}$ & $-93 b p$ & 1 & 0 & - \\
\hline g.106325876C > T & CT & $-56 b p$ & 1 & 1 & 1.000 \\
\hline g.106325849G > A & GA & $-30 b p$ & 1 & 0 & - \\
\hline g.106325757C > G (rs190825454) & GG & $+69 \mathrm{bp}$ & 0 & 1 & - \\
\hline
\end{tabular}

${ }^{1}$ Genetic variants are located upstream (-) to the transcription start site of the ATG5 gene at 106325820 of NC_000006.12.

[g.106325757C > G (rs190825454)] were only found in one 66-year-old male patient with non-ST-segment elevated AMI, and the sequencing chromatograms of which are shown in Figure 2(a). The SNP [g.106325757C > G (rs190825454)] was located in the untranslated exon 1 of the ATG5 gene. Two novel genetic variants (g.106325913T > C and g.106325849G > A) and three SNPs [g.106326548G > T (rs117781908), g.106326543G > T (rs182877945), and
g.106326293G > T (rs560549742)] were only identified in controls. The sequencing chromatograms of the two novel variants are shown in Figure 2(b). Six novel variants (g.106326740A > G, g.106326584A > T, g.106326487G > A, g.106326427_28insA, g.106326331G>A, and g.106325876C $>$ T) and two SNPs [g.106326589G $>$ A (rs506027) and g.106326155T > C (rs510432)] were found in both AMI patients and controls with similar frequencies. 
g.106326168_70delTCT

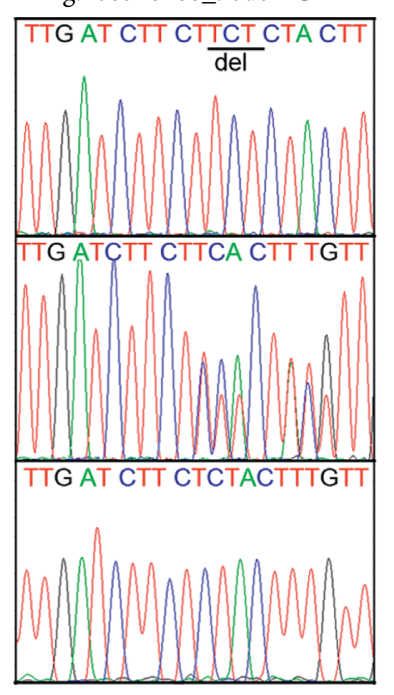

g.106325757C >G

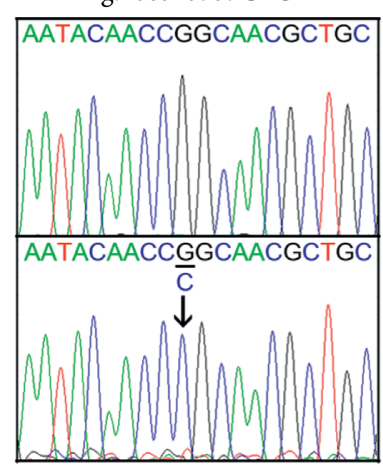

g.106325913T $>C$

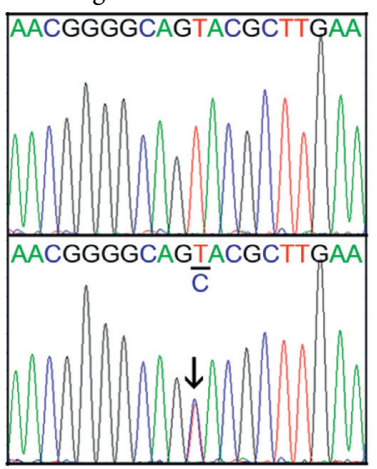

g.106325849G $>\mathrm{A}$

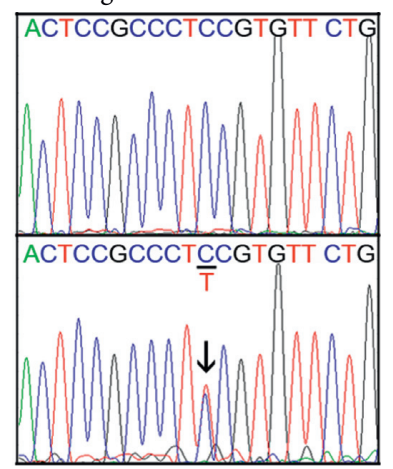

(a)

(b)

FIgURE 2: Sequencing chromatograms of the genetic variants in the ATG5 gene promoter. (a) Genetic variants in AMI patients. (b) Novel genetic variants in controls. For the variant (g.106326168_70delTCT), the top panel shows wild type, middle heterozygous, and bottom sequencing after cloning into a vector. For other genetic variants, top panels show wild-type DNA sequences and bottom panels show heterozygous variants, which are marked with arrows. For all variants, sequence orientation is forward.

Interestingly, two SNPs [g.106326589G > A (rs506027) and g.106326155T > C (rs510432)] were closely linked together with the same frequencies $(P>0.05)$.

3.1.1. Genetic Variant-Related Putative Binding Sites for Transcription Factors. To determine whether genetic variant identified in AMI patients affected putative biding sites for transcription factors, the ATG5 gene promoter was analyzed with the JASPAR program (http://jaspar.genereg.net/). The variant g.106326168_70delTCT was predicted to abolish the binding sites for the Spi-B transcription factor (SPIB) and GATA binding protein 6 (GATA6), create the binding sites for the homeodomain factor RAX and transcription factor 7like 2 (TCF7L2), and modify the binding sites for GATA2 and GATA3. As the SNP [g.106325757C > G (rs190825454)] was located in the untranslated exon 1 of the ATG5 gene, it was not analyzed.

3.2. Functional Analysis of the DSVs by Dual-Luciferase Reporter Assay. Activity of the wild-type and variant ATG5 gene promoter was examined in cultured cells by the dualluciferase reporter assay (Figure 3). Expression vectors included empty pGL3-basic (negative control), pGL3-WT (the wild-type ATG5 gene promoter), pGL3106326168_70delTCT, pGL3-g.106326293T, pGL3106325913C, pGL3-106325876T, and pGL3-106326155C. In both HEK-293 and H9c2 cells, deletion variant (g.106326168_70delTCT) found in an AMI patient significantly decreased the activity of the ATG5 gene promoter $(P<0.01)$. In contrast, the SNP [g.106326293G $>\mathrm{T}$ (rs560549742)] and genetic variant (g.106325913T $>C$ ), which were identified in controls, did not significantly affect the activity of the ATG5 gene promoter in both HEK-293 and $\mathrm{H} 9 \mathrm{c} 2$ cells $(P>0.05)$. As expected, the SNP [g.106326155T $>$ C (rs510432)] and genetic variant (g.106325876C > T), which were found in both AMI patients and controls, did not significantly alter the activity of the ATG5 gene promoter $(P>0.05)$. Collectively, the deletion genetic variant (g.106326168_70delTCT) may change the transcriptional activity of the ATG5 gene promoter, which was not tissue-specific. In addition, as the SNP [g.106325757C > G (rs190825454)] was located in the untranslated exon 1 of the ATG5 gene, its effect on the ATG5 gene promoter was not examined.

3.2.1. The Binding for Transcription Factors Affected by Genetic Variants. To experimentally confirm whether the deletion genetic variant (g.106326168_70delTCT) affected the binding sites for transcription factors, EMSA was performed with nuclear extracts from HEK-293 cells and H9c2 cells. Double-stranded biotinylated oligonucleotides (30 bp) included wild-type (5'-TCCAACAAAGTAGAGAAGAAGATCAAATAA- $3^{\prime}$ ) and genetic variant g.106326168_70delTCT (5'-TCCAACAAAGTAGAGAAGATCAAATAAGAA- $3^{\prime}$ ). The results showed that the deletion genetic variant evidently weakened the binding of a transcription factor in both HEK-293 and H9c2 cells. Combined with the data predicted by the JASPAR program, further work will be needed to identify the transcription factor (Figure 4).

\section{Discussion}

Genetic variants in the ATG5 gene have been associated with several human diseases. An intron SNP in the ATG5 gene (rs9373839) has been associated with systemic sclerosis [24]. 


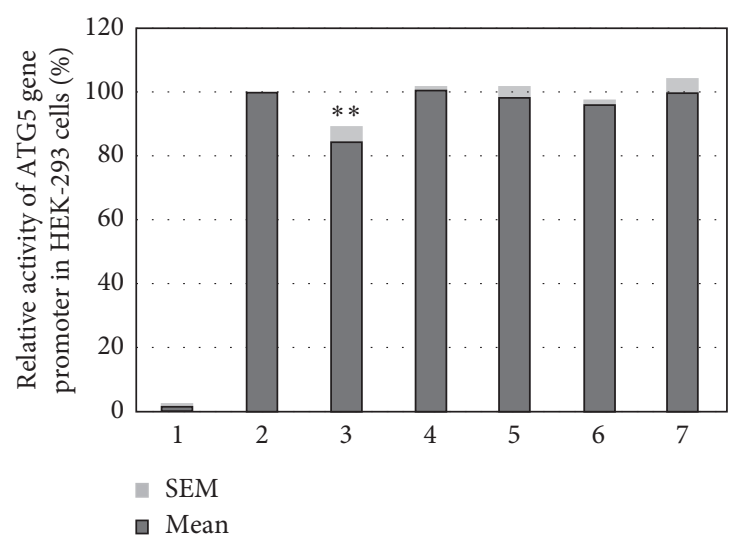

(a)

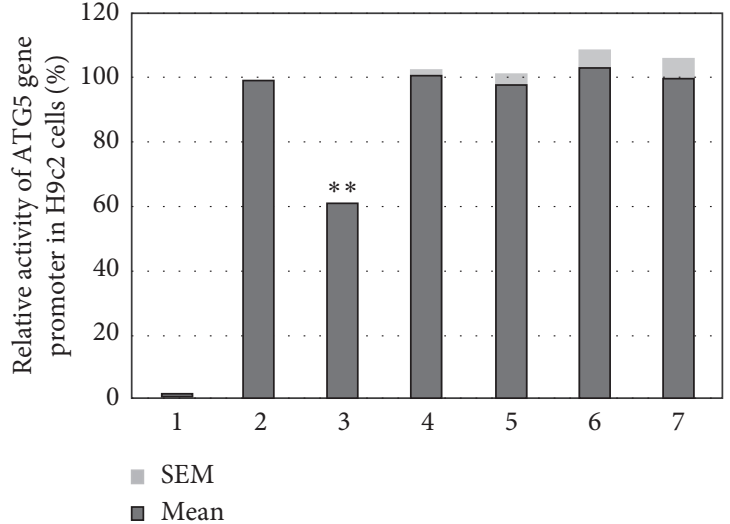

(b)

FIGURE 3: Relative transcriptional activity of wild-type and variant ATG5 gene promoters. Wild-type and variant ATG5 gene promoters were cloned into the reporter gene vector pGL3 and transfected into cultured cells. The transfected cells were collected, and dual-luciferase activities were assayed. Empty vector pGL3-basic was used as a negative control. Transcriptional acitivity of the wild-type ATG5 gene promoter was designed as 100\%. Relative activities of ATG5 gene promoters were calculated. (a). Relative activities of wild-type and variant ATG5 gene promoters in HEK-293 cells. (b). Relative activities of wild-type and variant ATG5 gene promoters in H9c2 cells. Lanes 1, pGL3basic; 2, pGL3-WT; 3, pGL3-106326168_70delTCT; 4, pGL3-g.106326293T; 5, pGL3-106325913C; 6, pGL3-106325876T, and 7, pGL3106326155C. ${ }^{* *}, P<0.01$.

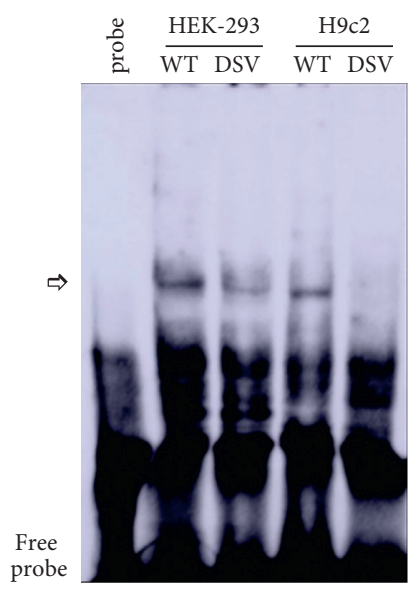

FIgURE 4: EMSA of biotin-labeled oligonucleotides containing the variant (g.106326168_70delTCT). Wild-type and variant oligonucleotides (30 bp) were designed and labeled with biotin. EMSA was conducted with biotinylated oligonucleotides and the nuclear extracts from HEK-293 and H9c2 cells. The free probe was marked with an arrow at the bottom. The affected binding for the transcription factor was marked with an open arrow.

An intron SNP (rs12201458) and a promoter SNP (rs510432) are implicated in childhood asthma [25]. Intron SNPs in the ATG5 gene (rs573775 and rs665791) and the SNPs in the PRDM1-ATG5 intergenic region (rs548234 and rs6937876) confer to the susceptibility of developing systemic lupus erythematosus in different populations [26-29]. An intron SNP in the ATG5 gene (rs9372120) has been associated to multiple myeloma by a genome-wide association study [30]. In this study, we found a deletion genetic variant in the ATG5 gene promoter (g.106326168_70delTCT) and an SNP [g.106325757C > G (rs190825454)] in untranslated exon 1 of the ATG5 gene in one AMI patient. Furthermore, the deletion genetic variant significantly decreased the transcriptional activity of the ATG5 gene promoter by affecting the binding of a transcription factor. Therefore, the genetic variant in the ATG5 gene promoter may affect its transcription activity and change ATG5 levels, contributing to the AMI development as a rare risk factor.

The human ATG5 gene has been mapped to 6q21 [31]. It is transcribed into the mRNAs of $3.3 \mathrm{~kb}, 2.5 \mathrm{~kb}$, and $1.8 \mathrm{~kb}$ at comparable levels in viable cells [32]. Though the ATG5 gene promoter has not been characterized, studies have demonstrated that the human ATG5 gene is regulated by transcription factors, microRNAs, and long noncoding RNAs. For example, P73 binds to and activates the human ATG5 gene promoter and regulates hepatocellular lipid metabolism [33]. NFE2L2 (nuclear factor, erythroid 2-like 2) activates ATG5 gene expression by regulating an enhancer antioxidant response element [34]. WNT5A overexpression leads to a significant increase in ATG5 mRNA levels in human melanoma cells [35]. miR-181A regulates the ATG5 gene through a functional mi-R181A responsive sequences in ATG5 $3^{\prime}$ UTR in human cells [36]. In hepatocellular carcinoma, ATG5 is a direct target of miR-30b and an indirect target of long noncoding RNA HNF1A-AS1 [37]. Knockdown of lncRNA DICER1-AS1 inhibits ATG5 protein levels in human osteosarcoma cells [38]. Altered levels of ATG5 have been associated with human diseases, including multiple sclerosis, Alzheimer's disease, and cancers [39-41]. In this study, the deletion genetic variant (g.106326168_70delTCT) may change the activity of the ATG5 gene promoter by modifying the binding of a transcription factor.

ATG5 is a critical core protein for autophagosome formation and autophagic function [11]. In mice with ATG5 gene deletion, defective autophagy is observed in different tissues [15]. Moreover, tissue-specific ATG5 deficiency in 
cardiomyocytes or neural cells in mice causes tissue-specific autophagy loss and suppression $[16,17,42]$. Overexpression of the ATG5 gene in mice activates autophagy [18]. Accumulating evidence has demonstrated that ATG5-dependent autophagy regulates lipid metabolism and inflammation $[4-6,19,43]$. Recent studies have revealed many nonautophagic functions of ATG5, including autophagy-dependent cell death, cell proliferation, exosome secretion, granule exocytosis, immunological memory, LC3-associated phagocytosis, no-canonical protein secretion, pathogen control, and vision cycle $[44,45]$. Particularly, LC3-associated phagocytosis as a novel function for autophagy proteins contributes to immune regulation and inflammatory responses $[46,47]$. ATG5 regulates secretion of mature interleukin 1 beta and prevents its degradation [48, 49]. Collectively, the altered ATG5 level may influence lipid metabolism and inflammation through its autophagic and nonautophagic function, contributing to atherosclerosis and its complications.

\section{Conclusions}

In this study, we found a novel and functional deletion genetic variant in the ATG5 gene promoter in an AMI patient, but in none of controls. The genetic variant significantly altered the transcriptional activity of the ATG5 gene promoter likely by affecting the binding of a transcription factor. Therefore, this genetic variant may change the ATG5 level, contributing to AMI development as a rare risk factor.

\section{Data Availability}

The data used to support the findings of this study are available from the corresponding author upon request.

\section{Conflicts of Interest}

The authors declare that there are no conflicts of interest regarding the publication of this paper.

\section{Authors' Contributions}

Yexin Zhang and Xiaohui He contributed equally to this work.

\section{Acknowledgments}

This study was supported by the National Natural Science Foundation of China (81370271, 81400291, and 81870279) and the Shandong Taishan Scholar Project, China (tshw201502063).

\section{References}

[1] G. R. Geovanini and P. Libby, "Atherosclerosis and inflammation: overview and updates," Clinical Science, vol. 132, no. 12, pp. 1243-1252, 2018.

[2] M. D. Shapiro and S. Fazio, "From lipids to inflammation," Circulation Research, vol. 118, no. 4, pp. 732-749, 2016.
[3] K. Musunuru and S. Kathiresan, "Genetics of common, complex coronary artery disease," Cell, vol. 177, no. 1, pp. 132-145, 2019.

[4] Y. Matsuzawa-Ishimoto, S. Hwang, and K. Cadwell, "Autophagy and inflammation," Annual Review of Immunology, vol. 36, no. 1, pp. 73-101, 2018.

[5] H. Morishita and N. Mizushima, "Diverse cellular roles of autophagy," Annual Review of Cell and Developmental Biology, vol. 35, no. 1, pp. 453-475, 2019.

[6] X. Zhang, T. D. Evans, S.-J. Jeong, and B. Razani, "Classical and alternative roles for autophagy in lipid metabolism," Current Opinion in Lipidology, vol. 29, no. 3, pp. 203-211, 2018.

[7] J. M. Bravo-San Pedro, G. Kroemer, and L. Galluzzi, "Autophagy and mitophagy in cardiovascular disease," Circulation Research, vol. 120, no. 11, pp. 1812-1824, 2017.

[8] A. M. K. Choi, S. W. Ryter, and B. Levine, "Autophagy in human health and disease," New England Journal of Medicine, vol. 368, no. 7, pp. 651-662, 2013.

[9] S. Sciarretta, Y. Maejima, D. Zablocki, and J. Sadoshima, "The role of autophagy in the heart," Annual Review of Physiology, vol. 80 , no. 1 , pp. 1-26, 2018.

[10] D. Gatica, M. Chiong, S. Lavandero, and D. J. Klionsky, "Molecular mechanisms of autophagy in the cardiovascular system," Circulation Research, vol. 116, no. 3, pp. 456-467, 2015.

[11] N. Mizushima, T. Yoshimori, and Y. Ohsumi, "The role of Atg proteins in autophagosome formation," Annual Review of Cell and Developmental Biology, vol. 27, no. 1, pp. 107-132, 2011.

[12] C. F. Bento, M. Renna, G. Ghislat et al., "Mammalian autophagy: how does it work?" Annual Review of Biochemistry, vol. 85, no. 1, pp. 685-713, 2016.

[13] N. N. Noda and F. Inagaki, "Mechanisms of autophagy," Annual Review of Biophysics, vol. 44, no. 1, pp. 101-122, 2015.

[14] L. Yu, Y. Chen, and S. A. Tooze, "Autophagy pathway: cellular and molecular mechanisms," Autophagy, vol. 14, no. 2, pp. $207-215,2018$.

[15] A. Kuma, M. Hatano, M. Matsui et al., "The role of autophagy during the early neonatal starvation period," Nature, vol. 432, no. 7020, pp. 1032-1036, 2004.

[16] A. Nakai, O. Yamaguchi, T. Takeda et al., "The role of autophagy in cardiomyocytes in the basal state and in response to hemodynamic stress," Nature Medicine, vol. 13, no. 5, pp. 619-624, 2007.

[17] M. Taneike, O. Yamaguchi, A. Nakai et al., "Inhibition of autophagy in the heart induces age-related cardiomyopathy," Autophagy, vol. 6, no. 5, pp. 600-606, 2010.

[18] J.-O. Pyo, S.-M. Yoo, H.-H. Ahn et al., "Overexpression of Atg5 in mice activates autophagy and extends lifespan," Nature Communications, vol. 4, no. 1, p. 2300, 2013.

[19] W. Zhao, Y. Li, L. Jia, L. Pan, H. Li, and J. Du, "Atg5 deficiency-mediated mitophagy aggravates cardiac inflammation and injury in response to angiotensin II," Free Radical Biology and Medicine, vol. 69, pp. 108-115, 2014.

[20] I. Sergin, S. Bhattacharya, R. Emanuel et al., "Inclusion bodies enriched for p62 and polyubiquitinated proteins in macrophages protect against atherosclerosis," Science Signaling, vol. 9, no. 409, 2016.

[21] G. Wu, G. Wei, J. Huang, S. Pang, L. Liu, and B. Yan, "Decreased gene expression of LC3 in peripheral leucocytes of patients with coronary artery disease," European Journal of Clinical Investigation, vol. 41, no. 9, pp. 958-963, 2011.

[22] F. Gao, Q. Su, W. Yang et al., "Functional variants in the LC3B gene promoter in acute myocardial infarction," Journal of Cellular Biochemistry, vol. 119, no. 9, pp. 7339-7349, 2018. 
[23] P. Zhang, J. Zhang, Y. Zhang, S. Wang, S. Pang, and B. Yan, "Functional variants of the ATG7 gene promoter in acute myocardial infarction," Molecular Genetics \& Genomic Medicine, vol. 6, no. 6, pp. 1209-1219, 2018.

[24] M. D. Mayes, L. Bossini-Castillo, O. Gorlova et al., "Immunochip analysis identifies multiple susceptibility loci for systemic sclerosis," The American Journal of Human Genetics, vol. 94, no. 1, pp. 47-61, 2014.

[25] L. J. Martin, J. Gupta, S. S. S. K. Jyothula et al., "Functional variant in the autophagy-related 5 gene promotor is associated with childhood asthma," PLoS One, vol. 7, no. 4, Article ID e33454, 2012.

[26] C. Ciccacci, C. Perricone, C. Alessandri et al., "Evaluation of ATG5 polymorphisms in Italian patients with systemic lupus erythematosus: contribution to disease susceptibility and clinical phenotypes," Lupus, vol. 27, no. 9, pp. 1464-1469, 2018.

[27] J. Dang, J. Li, Q. Xin et al., "Gene-gene interaction of ATG5, ATG7, BLK and BANK1 in systemic lupus erythematosus," International Journal of Rheumatic Diseases, vol. 19, no. 12, pp. 1284-1293, 2016.

[28] P. López, E. Alonso-Pérez, J. Rodríguez-Carrio, and A. Suárez, "Influence of Atg5 mutation in SLE depends on functional IL10 genotype," PLoS One, vol. 8, no. 10, Article ID e78756, 2013.

[29] X.-j. Zhou, X.-1. Lu, J.-c. Lv et al., "Genetic association of PRDM1-ATG5 intergenic region and autophagy with systemic lupus erythematosus in a Chinese population," Annals of the Rheumatic Diseases, vol. 70, no. 7, pp. 1330-1337, 2011.

[30] J. S. Mitchell, N. Li, N. Weinhold, A. Försti et al., "Genomewide association study identifies multiple susceptibility loci for multiple myeloma," Nature Communications, vol. 7, p. 12050, 2016.

[31] K. Schmeiser, S. Armstrong, E. M. Hammond, and R. J. Grand, "Assignment of the yeast APG5 human homologue APG5L to chromosome band 6q21 by fluorescence in situ hybridisation," Cytogenetic and Genome Research, vol. 87, no. 3-4, pp. 213-214, 1999.

[32] E. M. Hammond, C. L. Brunet, G. D. Johnson et al., "Homology between a human apoptosis specific protein and the product of APG5, a gene involved in autophagy in yeast," FEBS Letters, vol. 425, no. 3, pp. 391-395, 1998.

[33] Z. He, H. Liu, M. Agostini et al., "p73 regulates autophagy and hepatocellular lipid metabolism through a transcriptional activation of the ATG5 gene," Cell Death \& Differentiation, vol. 20, no. 10, pp. 1415-1424, 2013.

[34] M. Pajares, N. Jiménez-Moreno, Á. J. García-Yagüe et al., "Transcription factor NFE2L2/NRF2 is a regulator of macroautophagy genes," Autophagy, vol. 12, no. 10, pp. 19021916, 2016.

[35] A. Ndoye, A. Budina-Kolomets, C. H. Kugel et al., "ATG5 mediates a positive feedback loop between wnt signaling and autophagy in melanoma," Cancer Research, vol. 77, no. 21, pp. 5873-5885, 2017.

[36] K. A. Tekirdag, G. Korkmaz, D. G. Ozturk, R. Agami, and D. Gozuacik, "MIR181Aregulates starvation- and rapamycininduced autophagy through targeting of ATG5," Autophagy, vol. 9, no. 3, pp. 374-385, 2013.

[37] Z. Liu, X. Wei, A. Zhang, C. Li, J. Bai, and J. Dong, "Long noncoding RNA HNF1A-AS1 functioned as an oncogene and autophagy promoter in hepatocellular carcinoma through sponging hsa-miR-30b-5p," Biochemical and Biophysical Research Communications, vol. 473, no. 4, pp. 1268-1275, 2016.
[38] Z. Gu, Z. Hou, L. Zheng, X. Wang, L. Wu, and C. Zhang, "LncRNA DICER1-AS1 promotes the proliferation, invasion and autophagy of osteosarcoma cells via miR-30b/ATG5," Biomedicine \& Pharmacotherapy, vol. 104, pp. 110-118, 2018.

[39] M. Alirezaei, H. S. Fox, C. T. Flynn et al., "Elevated ATG5 expression in autoimmune demyelination and multiple sclerosis," Autophagy, vol. 5, no. 2, pp. 152-158, 2009.

[40] S.-J. Cho, H. J. Lim, C. Jo, M. H. Park, C. Han, and Y. H. Koh, "Plasma ATG5 is increased in Alzheimer's disease," Scientific Reports, vol. 9, no. 1, p. 4741, 2019.

[41] K. Görgülü, K. N. Diakopoulos, J. Ai et al., "Levels of the autophagy-related 5 protein affect progression and metastasis of pancreatic tumors in mice," Gastroenterology, vol. 156, no. 1, pp. 203-217, 2019.

[42] T. Hara, K. Nakamura, M. Matsui et al., "Suppression of basal autophagy in neural cells causes neurodegenerative disease in mice," Nature, vol. 441, no. 7095, pp. 885-889, 2006.

[43] K. N. Diakopoulos, M. Lesina, S. Wörmann et al., "Impaired autophagy induces chronic atrophic pancreatitis in mice via sex- and nutrition-dependent processes," Gastroenterology, vol. 148, no. 3, pp. 626-638, 2015.

[44] L. Galluzzi and D. R. Green, "Autophagy-independent functions of the autophagy machinery," Cell, vol. 177, no. 7, pp. 1682-1699, 2019.

[45] S. Subramani and V. Malhotra, "Non-autophagic roles of autophagy-related proteins," EMBO Reports, vol. 14, no. 2, pp. 143-151, 2013.

[46] B. L. Heckmann and D. R. Green, "LC3-associated phagocytosis at a glance," Journal of Cell Science, vol. 132, no. 5, p. jcs222984, 2019.

[47] M. A. Sanjuan, C. P. Dillon, S. W. G. Tait et al., "Toll-like receptor signalling in macrophages links the autophagy pathway to phagocytosis," Nature, vol. 450, no. 7173, pp. 1253-1257, 2007.

[48] N. Dupont, S. Jiang, M. Pilli, W. Ornatowski, D. Bhattacharya, and V. Deretic, "Autophagy-based unconventional secretory pathway for extracellular delivery of IL-1 $\beta$," The EMBO Journal, vol. 30, no. 23, pp. 4701-4711, 2011.

[49] H. Guo, M. Chitiprolu, L. Roncevic et al., "Atg5 disassociates the V1V0-ATPase to promote exosome production and tumor metastasis independent of canonical macroautophagy," Developmental Cell, vol. 43, no. 6, pp. 716-730, 2017. 